\title{
The Pharmacological Basis of Altered Drug Response in the Elderly
}

\author{
Ian H. STEVENSON*
}

The clinical pharmacology of the elderly has been a subject of growing importance over the last fifteen years, ${ }^{1)}$ the following five areas of investigation receiving particular attention in relation to age.

- Frequency of adverse drug reactions

- Patterns of drug prescribing

- Compliance problems

- Alteration in pharmacokinetics

- Change in receptor sensitivity or density

As has been extensively reviewed, ${ }^{2-5)}$ there is now a considerable body of evidence to indicate that the response to drugs may change as people age, with many drugs the frequency of adverse drug reaction being significantly higher in the older age groups. ${ }^{6)}$ Multiple drug prescribing and difficulty in complying with prescribing instructions are major problems in this age group and, without doubt, contribute very significantly to the occurrence of adverse drug reactions. Even in the absence of such complications however, age-related differences in drug response occur for which alteration in drug disposition (pharmacokinetics) or change in the sensitivity or density of the receptors on which drugs act (pharmacodynamics) are likely to be responsible. A further complication in the elderly is a well-recognised but, in terms of mechanism, poorly-investigated impairment of the physiological homeostatic mechanisms which moderate drug effects.

Pharmacokinetic Processes in the Elderly

As people age, several physiological changes occur in the gastrointestinal tract (the principal site of drug absorption), in the liver (the principal

* Department of Pharmacology and Clinical Pharmacology, Ninewells Hospital and Medical School

Dundee, DD 19 SY organ of drug biotransformation or metabolism) and in the kidney (the principal organ involved in the excretion of drugs and their metabolites). In view of these changes it is not surprising that significant pharmacokinetic changes do occur in the elderly, the main aspects of which are summarised in Tab. 1.

\section{Drug Absorption}

The subject of drug absorption in the elderly has previously been reviewed. ${ }^{4,7)}$ Many of the early studies are of inadequate design in that they do not compare intravenous and oral plasma drug concentration versus time profiles in the two age groups.

Changes, where they do occur, are often small and unlikely to be of clinical significance. With digoxin, for example, the extent of absorption does not change significantly with age and the only difference occurring in older patients is a delay in time to reach peak plasma concentrations. The only drug for which there is evidence of a substantial reduction in extent of absorption, in the absence of gastrointestinal pathology, is prazosin, the systemic availability of which in the elderly is only some $60 \%$ of that in younger subjects. $^{8)}$ Age-related changes in the first-pass extraction of some drugs are important and will be referred to in the metabolism section.

\section{Drug Distribution}

Major changes in drug distribution occur in old age due particularly to the marked reduction in lean body mass and the increase in body fat as fat replaces functional tissue. While it is difficult to generalise, the distribution of hydrophilic drugs such as antipyrine, quinine and paracetamol appears to decrease in the elderly whereas the distribution volumes of lipophilic drugs such as chlordiazepoxide and diazepam is increased, in some cases to an extent considerably greater than that 
Tab. 1 Physiological and Pharmacokinetic Changes Occurring in the Elderly*

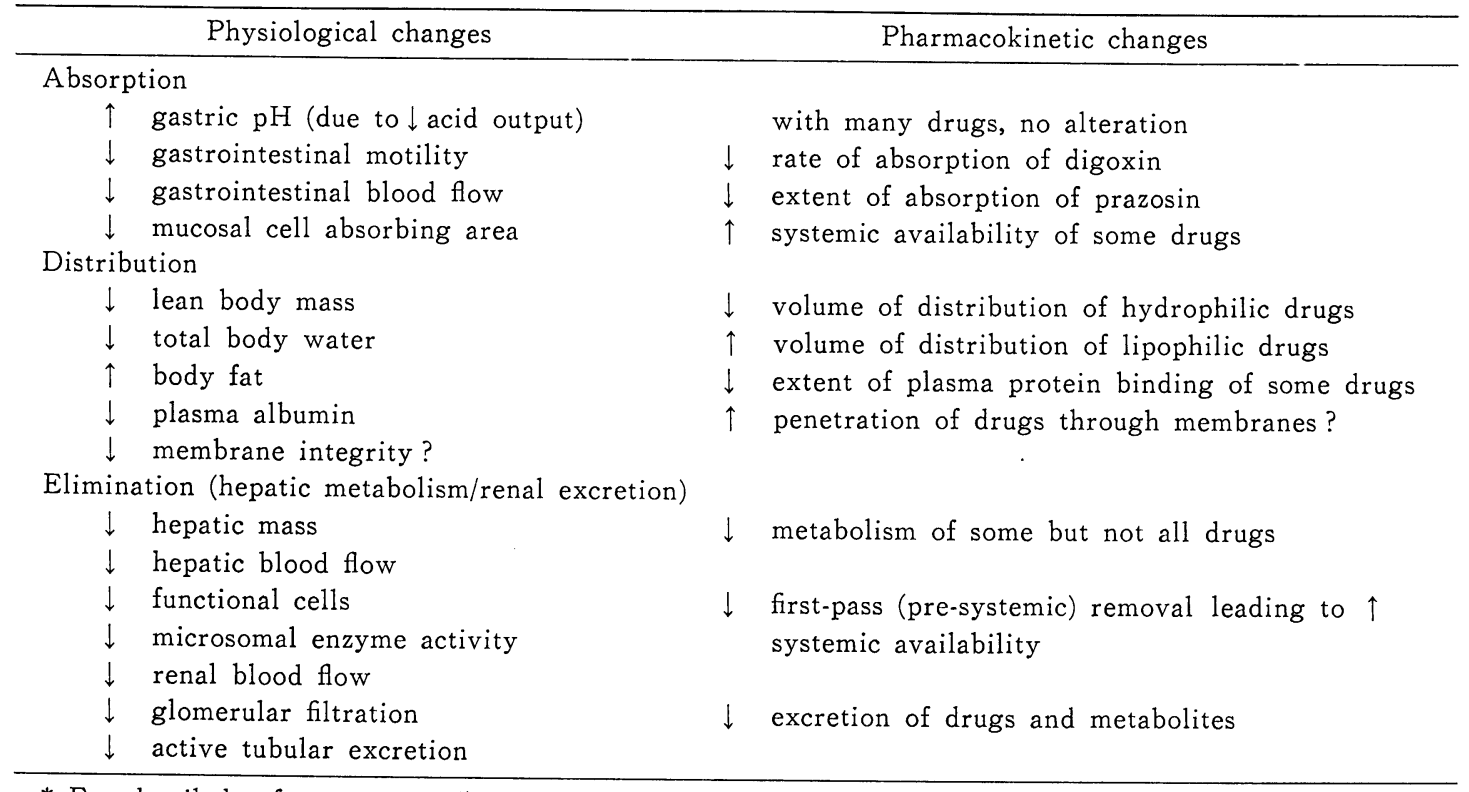

* For detailed references, see 4$)$.

predicted from physiological changes.

Plasma albumin concentrations are slightly lower in healthy old people and may be markedly so in the poorly nourished or severely debilitated elderly, resulting in an increase in the free pharmacologically-active fraction of some drugs and in turn leading to a greater distribution and a more rapid elimination. In healthy elderly subjects, however, changes in extent of plasma protein binding, where they occur, are small. There is also some evidence to suggest that, in the elderly drugs may be more readily displaced from plasma protein binding sites by other drugs. ${ }^{92}$

One aspect of distribution so far poorly investigated and about which little is known in relation to age is the penetration of drugs through membranes or through membrane like systems such as the blood brain barrier. Results from animal studies have so far been equivocal and there has been little or no opportunity so far to carry out such investigations in man. With the advent of Positron Emission Tomography however the possibility would now exist of carrying out age-related comparisons of the penetration of appropriatelylabelled drugs into the brain. Interestingly, in a recent investigation of thiopentone distribution and effect in patients, a sophisticated pharmacokinetic analysis indicated a marked age-related increase in the rate of transfer of thiopentone from the central compartment to both the rapidly and slowly equilibrating compartments. ${ }^{10)}$

\section{Elimination}

Drug elimination involves the two linked processes of hepatic metabolism (whereby most drugs are converted to more polar metabolites) and renal excretion (the excretion by the kidney of polar drugs and metabolites). The metabolism of many drugs, and particularly those oxidised by the hepatic microsomal, cytochrome P-450 dependent oxidases, is reduced in old age, partly because of the age-related decrease in functional liver volume and partly as a result of a reduction in microsomal enzyme activity. ${ }^{11}$ Many drugs undergoing oxidation show this reduced rate of metabolism e. g. antipyrine, chlordiazepoxide, propranolol and nortriptyline yet this is not a general effect since a few other drugs which are also oxidised e. g. warfarin, metoprolol and prazosin show no such change with ageing. The important conjugation process of glucuronide conjugation appears to be less affected by ageing and the metabolism of several benzodiazepines metabolised by this route is unchanged with ageing. ${ }^{12)}$

Changes in drug metabolism with age, where 
they occur, are often small and may fall within the range of inter-individual variability in a healthy younger population. In a large study on antipyrine, for example, in a population ranging in age from approximately 20 to 90 , Vestal ${ }^{13}$ ) demonstrated that less than $10 \%$ of the variability in antipyrine clearance was due to age, that environmental factors such as smoking were also important and that much of the variance in clearance remained unexplained.

A number of drugs are so avidly extracted by the liver, i. e. by uptake into hepatic binding sites and by metabolism, that their clearance depends on rate of delivery to the liver in the blood. In old age, a decrease in hepatic blood flow together with possible reduction in the rate of hepatic metabolism is responsible for the reduced elimination of such high clearance drugs as chlormethiazole, ${ }^{14)}$ labetalol ${ }^{15)}$ and zimelidine. ${ }^{16)}$ With such highly-cleared drugs there is a marked first-pass effect due to their extensive pre-systemic removal from the blood on their first passage through the liver. Their oral bioavailability is therefore low but is increased in the elderly because of a reduction in the first pass extraction. Again however this is not a general phenomenon with high clearance drugs since no decrease in clearance occurs with either lignocaine ${ }^{17)}$ or prazosin. ${ }^{8}{ }^{8}$ With another high clearance drug, propranolol, the evidence is conflicting although in studies on hyperthyroid patients receiving this drug prior to thyroidectomy, steady-state levels increased with age of patient and this relationship held even in the presence of complicating factors such as smoking habit and severity of thyroid disease. ${ }^{18)}$

A complicating factor in many studies on drug metabolism in the elderly is the presence of disease or hospitalisation of the population under study. Swift et al. ${ }^{11)}$ drew attention to this in relation to antipyrine clearance. Patient population differences have also been found in the authors' laboratory in relation to the elimination of the anti-inflammatory drug benoxaprofen in elderly patients (Tab. 2). The elimination half-life in our study in arthritic outpatients (i. e. relatively healthy elderly patients living at home) is very much closer to the value for healthy young subjects than those reported by Hamdy et al. and by Kamal et al. for hospitalised elderly patients. The results would therefore suggest that, based on half-life data, the elimination of this drug is less influenced by age per se than by hospitalisation and the factors associated with this, principally severity of illness.

In an extension of our work in these patients the plasma elimination half-life of benoxaprofen on stopping therapy after several months was not significantly different from the initial dosing of benoxaprofen occurred during this period above the steady-state levels predicted from the initial dosing half-life.

The effects of age on renal function exert a profound influence on the elimination of a number of drugs. ${ }^{2,3,6)}$ In many cases drugs are excreted by simple glomerular filtration and their rate of excretion correlates with glomerular filtration rate and hence with creatinine clearance, e. g. digoxin and the aminoglycoside antibiotics. In old age, renal function diminishes partly as a result of reduced renal blood flow, so that at age 65 there is a reduction of approximately $30 \%$ in glomerular filtration rate compared with young adults. Tubular function also deteriorates with age and drugs such as penicillin and procainamide which are actively secreted by renal tubules show a marked reduction in clearance. Whether age per se exerts such a marked influence on renal elimination of drugs is uncertain but renal drug clearance may be very much reduced in elderly patients.

Where there is obvious renal disease, guidance for appropriate dosage of drugs excreted unchanged in the urine may be obtained from standard nomograms. With some drugs this is particularly

Tab. 2 Benoxaprofen Elimination Kinetics

\begin{tabular}{lccl}
\hline Group studied & Age $(\mathrm{yr})$ & Elimination $\mathrm{t}_{1 / 2}(\mathrm{hr})$ & \multicolumn{1}{c}{ Study } \\
\hline Young male subjects $(\mathrm{n}=20)$ & $21-55$ & 25.7 & Nash et al. ${ }^{17)}$ \\
Female inpatients $(\mathrm{n}=6)$ & $81.8 \pm 1.6^{*}$ & $111(90-37)$ & Hamdy et al. ${ }^{19)}$ \\
Mixed sex inpatients $(\mathrm{n}=10)$ & $76.9 \pm 2.1^{*}$ & $101(54-325)$ & Kamal et al. $\left.{ }^{20}\right)$ \\
Mixed sex arthritic outpatients $(\mathrm{n}=9)$ & $60.9 \pm 0.84^{*}$ & $47.6(28.5-73.9)$ & Stevenson and Hosie \\
\hline
\end{tabular}

* Mean \pm SEM 
Tab. 3 Alterations in Drug Response in Old Age Occurring in the Absence of Pharmacokinetic Change

\begin{tabular}{llc}
\hline \multicolumn{1}{c}{ Drug } & \multicolumn{1}{c}{ Effect in old age } & Reference \\
\hline Warfarin & $\uparrow$ anticoagulation & $21,22)$ \\
Nitrazepam & $\uparrow$ impairment of performance & $23)$ \\
Diazepam & $\downarrow$ dose for CNS depression & $24)$ \\
Diazepam & $\downarrow$ dose for intubation & $25)$ \\
Diazepam & $\uparrow$ body sway & $26)$ \\
Temazepam & $\uparrow$ body sway and reaction time & $27)$ \\
& $\downarrow$ CNS arousal & $27)$ \\
Chlormethiazole & $\uparrow$ body sway and reaction time & $27)$ \\
& $\downarrow$ CNS arousal & $27)$ \\
Dichloralphenazone & $\uparrow$ body sway & $27)$ \\
Isoprenaline & $\uparrow$ dose for tachycardia & $28)$ \\
Propranolol & $\uparrow$ dose for block of isoprenaline induced tachycardia & $28)$ \\
Propranolol & $\uparrow$ dose for block of exercise induced tachycardia & $29)$ \\
\hline
\end{tabular}

important because of the serious effects of over dosage-e. g. digoxin, lithium and the aminoglycoside antibiotics. In general elderly patients are best treated with lower doses of such drugs than are younger patients.

\section{Pharmacodynamic Considerations in the Elderly}

Despite the presence of many differences in drug handling which help to account for variations in drug effects between the old and the young, there is still, as summarised in Tab. 3, a significant residue of altered responsiveness which seems to be explicable only by differences in tissue 'sensitivity' to drugs. It is obviously not possible in most cases to measure true receptor sensitivity and most studies simply relate drug effect to the dose required or to the plasma drug concentration at which the effect occurred. Shepherd et al. ${ }^{22}$ investigated the mechanism involved in the greater sensitivity of elderly patients to warfarin ${ }^{21)}$ and demonstrated that at the same plasma concentration, there was greater inhibition of Vitamin$\mathrm{K}$-dependent clotting factor synthesis in the elderly than in younger patients. The innovative studies of Castleden et al. $^{23}$ indicated an increased sensitivity of the elderly central nervous system to nitrazepam in that single doses impaired psychomotor performance function in the elderly to a greater extent than in the young, in the absence of any age-related pharmacokinetic differences. Similar findings have subsequently been reported for the hypnotics temazepam and chlormethiazole ${ }^{27)}$ but to a lesser extent for diazepam ${ }^{24)}$ and dichloralphenazone. ${ }^{27)}$ The elderly have also been reported to be more sensitive to the depressant effects of diazepam as indicated by the plasma concentration to achieve sedation in patients being premedicated for elective cardioversion ${ }^{24)}$ and by dose requirements for intubation in patients undergoing endoscopy. ${ }^{25)}$

Several studies have implicated alteration in $\beta$ adrenoreceptor sensitivity in old age. Vestal et al. ${ }^{28)}$ demonstrated an age-related increase in the dose of isoprenaline required to induce tachycardia and showed that the extent of blockade of the isoprenaline-induced tachycardia by propranolol was decreased in older healthy subjects. Confirmatory evidence of a reduced effect of propranolol in the elderly has come from studies in the author's department. ${ }^{18)}$ From their results, Vestal et al. $^{28)}$ hypothesised a reduction in the number of receptors with increasing age.

As previously referred to, elderly patients often show an exaggerated response to single doses of a number of benzodiazepine hypnotics and adverse effects might be anticipated with chronic use in view of the tendency of such drugs to accumulate in the elderly. Interestingly, in a community study by Swift et al. ${ }^{29)}$ no appreciable impairment of mental function was found in elderly patients on long-term therapy with nitrazepam or flurazepam. The findings indicate therefore that CNS adaptation to chronic benzodiazepine therapy may occur in the elderly.

Conclusion: A summary of the drugs liable to produce special problems in the elderly is given in Tab. 4. The response to many drugs undoubtedly changes as people age as a result of a complex 
Tab. 4 Drugs Liable to Produce Special Problems in the Elderly

1. Drugs intended for widespread use in elderly populations.

2. Drugs with a steep dose-response curve and low therapeutic ratio-particularly if:

(a) eliminated by renal mechanisms

(b) undergoes first-pass extraction

3. Psychoactive drugs

4. Drugs whose effects are likely to be modified by homeostatic mechanisms.

alteration, with ageing, of pharmacokinetic processes, sensitivity factors and physiological homeostatic mechanisms. Of the pharmacokinetic changes, decreased renal elimination and a reduced first pass extraction resulting in increased drug bioavailability are particularly important. In the case of tissue sensitivity, there have been few opportunities so far to determine age-related changes in the number and sensitivity of drug receptors and studies have been largely confined to relating drug effects to plasma levels in patients of different age.

Such investigations have been complicated by the presence of disease in the elderly populations under study and it is difficult to differentiate between age effects per se and those associated with disease. In the evaluation of new drugs it is essential to obtain a comprehensive data base from which drug regimens appropriate for elderly patients may be developed.

\section{References}

1) Friedel, R. O.: Gerontology, 28 (Suppl. 1) : 5-7 (1982).

2) Vestal, R. E. : Drugs, $16: 359-382$ (1978).

3) Crooks, J. and Stevenson, I. H. (Ed.): Drugs and the Elderly, Macmillan, London (1979).

4) Stevenson, I. H. : Lemberger, L. and Reidenberg, M. M. (Ed.) : Proceedings of the Second World Conference on Clinical Pharmacology and Therapeutics, pp. 64-73 (1984).

5) MacLennan, W. J., Shepherd, A. N. and Stevenson, I. H.: Treatment in Clinical Medicine (Series). Reid, J. L. (Ed.) : The Elderly, SpringerVerlag, Berlin (1984).

6) Koch-Weser, J., Greenblatt, D. J., Sellers, E. M., et al. : N. Engl. J. Med., 306 : 1081-1086 (1982).

7) Stevenson, I. H., Salem, S. A. M., O’Malley, K. et al. : Prescott, L. F. and Nimmo, W.S. (Ed.): Drug Absorption, Adis Press, New York, pp. 253-261 (1980).

8) Rubin, P. C., Scott, P. J. W. and Reid, J. L. : Br. J. Clin. Pharmacol., 12 : 401-405 (1981).

9) Wallace, S., Whiting, B. and Runcie, J.: Br. J. Clin. Pharmacol., 3 : 327-333 (1976).

10) Homer, T.D. and Stanski, D. R. : Anaesthesiology, 62 : 714-724 (1985).

11) Swift, C. G., Homeida, M., Halliwell, M. et al. : Eur. J. Clin. Pharmacol., 14 : 149-152 (1978).

12) Swift, C. G. and Stevenson, I. H. : Costa, E. (Ed.) : The Benzodiazepines-From Molecular Biology to Clinical Practice, Raven Press, New York, pp. 225-236 (1983).

13) Vestal, R.E., Norris, A. H., Tobin, J. D. et al. : Clin. Pharmacol. Therap., 18 : 425-432 (1975).

14) Nation, R, L., Vine, J., Triggs, E. J. et al. : Eur. J. Clin. Pharmacol., 12 : 137-146 (1977).

15) Kelly, J. G., McGarry, C., O’Malley, K. et al. : Br. J. Clin. Pharmacol., 14 : 304-305 (1982).

16) Swift, C. G., Haythorne, J. M., Clarke, P. et al. : Acta Psychiatrs. Scand., 63 : 425-432 (1981).

17) Nash, J.F., Carmichael, R. H., Ridolfo, A. S. et al. : J. Rheumatol., 7 : 5-11 (1980).

18) Feely, J. and Stevenson, I. H.: J. Clin. Exptl. Gerontol., 1 : 173-184 (1979).

19) Hamdy, R. C., Murnane, B., Perera, N., et al.: Eur. J. Rheumatol. Inflamm., 5 : 69-75 (1982).

20) Kamal, Koch, I. M. : Eur. J. Rheumatol. Inflamm., 5 : 76-81 (1982).

21) O'Malley, K., Stevenson, I. H., Ward, C. et al. : Br. J. Clin. Pharmac., 4 : 309-314 (1977).

22) Shepherd, A. M. M., Hewick, D.S., Moreland, T. A. et al.: Br. J. Clin. Pharmac., 4 : 315-320 (1977).

23) Castleden, C. M., George, C.F., Marcer, D. et al. : Br. Med. J., 1 : 10-12 (1977).

24) Reidenberg, M. M., Levy, M., Warner, H. et al. : Clin. Pharmac. Ther., 23 : 371-374 (1978).

25) Giles, H. G., Macleod, S. M., Wright, J. R. et al. : Can. Med. Assoc. J., 118 : 513-514 (1978).

26) Swift, C. G. and Stevenson, I. H.: Benzodiazepines in the elderly. Costa, E. (Ed.): The Benzodiazepines, Raven Press, New York, pp. 225236 (1983).

27) Stevenson, I. H., Hockings, N.F. and Swift, C. G. : Kitani, K. (Ed.): Liver and Aging, Elsevier, Amsterdam, pp. 317-328 (1986).

28) Vestal, R.E., Wood, A. J. J. and Shand, D. G. : Clin. Pharmac. Ther., 26 : 181-186 (1979).

29) Swift, C. G., Swift, M. R., Hamley, J. et al. : Age and Aging, 13 : 335-343 (1984). 